\title{
Restricted frame graphs and a conjecture of Scott
}

\author{
Jérémie Chalopin* \\ LIF, CNRS \& Univ. Aix-Marseille \\ Marseille, France \\ jeremie.chalopin@lif.univ-mrs.fr \\ Zhentao Li \\ Département d'Informatique \\ École Normale Supérieure \\ Paris, France \\ zhentao.li@ens.fr
}

\author{
Louis Esperet ${ }^{\dagger}$ \\ G-SCOP, CNRS \& Univ. Grenoble Alpes \\ Grenoble, France \\ louis.esperet@g-scop.fr \\ Patrice Ossona de Mendez $z^{\ddagger}$ \\ CAMS, CNRS \& \\ École des Hautes Études en Sciences Sociales \\ Paris, France \\ IUUK, Charles University \\ Prague, Czech Republic \\ pom@ehess.fr
}

Submitted: Jun 2, 2014; Accepted: Feb 2, 2016; Published: Feb 19, 2016

Mathematics Subject Classifications: 05C17, 05C75, 05C62

\begin{abstract}
Scott proved in 1997 that for any tree $T$, every graph with bounded clique number which does not contain any subdivision of $T$ as an induced subgraph has bounded chromatic number. Scott also conjectured that the same should hold if $T$ is replaced by any graph $H$. Pawlik et al. recently constructed a family of trianglefree intersection graphs of segments in the plane with unbounded chromatic number (thereby disproving an old conjecture of Erdős). This shows that Scott's conjecture is false whenever $H$ is obtained from a non-planar graph by subdividing every edge at least once.

It remains interesting to decide which graphs $H$ satisfy Scott's conjecture and which do not. In this paper, we study the construction of Pawlik et al. in more details to extract more counterexamples to Scott's conjecture. For example, we show that Scott's conjecture is false for any graph obtained from $K_{4}$ by subdividing every edge at least once. We also prove that if $G$ is a 2-connected multigraph with no vertex contained in every cycle of $G$, then any graph obtained from $G$ by subdividing every edge at least twice is a counterexample to Scott's conjecture.
\end{abstract}

\footnotetext{
*Partially supported by ANR Project MACARON (ANR-13-JS02-0002).

${ }^{\dagger}$ Partially supported by ANR Project Heredia (ANR-10-JCJC-0204-01), ANR Project Stint (ANR-13BS02-0007), and LabEx PERSYVAL-Lab (ANR-11-LABX-0025-01).

${ }^{\ddagger}$ Partially supported by grant ERCCZ LL-1201, by the European Associated Laboratory "Structures in Combinatorics" (LEA STRUCO), and by ANR Project Stint ANR-13-BS02-0007.
} 


\section{Introduction}

A class of graph is $\chi$-bounded if there is a function $f$ such that every graph $G$ in the class satisfies $\chi(G) \leqslant f(\omega(G))$, where $\chi(G)$ is the chromatic number and $\omega(G)$ is the clique number of $G$. It is well known that the class of all graphs is not $\chi$-bounded [8].

Gyárfás [4] (see also [5]) conjectured that for any tree $T$, the class of graphs that do not contain $T$ as an induced subgraph is $\chi$-bounded. This conjecture is still open, but Scott proved the following topological variant in 1997 [11]: for any tree $T$, the class of graphs that do not contain any subdivision of $T$ as an induced subgraph is $\chi$-bounded. Scott conjectured that the same property should hold whether $T$ is a tree or not. On the other hand, it is easy to see that Gyárfás's conjecture is false if $T$ contains a cycle as there are graphs of arbitrarily high girth (ensuring no copy of $T$ appears) and high chromatic number [3].

Conjecture 1.1 (Scott's conjecture [11]). For any $H$, the class of graphs excluding all subdivisions of $H$ as an induced subgraph is $\chi$-bounded.

$\mathrm{A} \geqslant k$-subdivision of a (multi)graph $G$ is a graph obtained from $G$ by subdividing each edge at least $k$ times, i.e. replacing every edge of $G$ by a path on at least $k+1$ edges. A recent result [10] (see [9] for a follow-up) shows that Scott's conjecture is false whenever $H$ is a $\geqslant 1$-subdivision of a non-planar graph. The proof is based on a construction of a family of triangle-free intersection graphs of segments in the plane with unbounded chromatic number (the existence of such graphs also disproved a conjecture of Erdös). Since no $\geqslant 1$-subdivision of a non-planar graph can be represented as the intersection of arcwise connected sets in the plane (in particular, such subdivisions cannot be represented as an intersection graph of line segments) no such graph appears as an induced subgraph in the construction. Therefore, graphs in the construction exclude all subdivisions of 1subdivisions of non-planar graphs as induced subgraphs. Hence, the 1-subdivision of any non-planar graph is a counterexample to Scott's conjecture.

Recently, Walczak [13] showed how to slightly modify the construction of $[10,9]$ to obtain a family of graphs with no stable sets of linear size (in particular, with unbounded fractional chromatic number). Therefore, the following stronger result can be deduced: for any non-planar graph $H$, there exist graphs with no $\geqslant 1$-subdivision of $H$ as an induced subgraph, and with no stable sets of linear size.

Note that the construction of $[10,9]$ gives the same family of graphs as a construction of Burling [1], who proved in 1965 that triangle-free intersection graphs of axis-parallel boxes in $\mathbb{R}^{3}$ have unbounded chromatic number.

\section{Our results}

Our original goal was to characterize all graphs $H$ such that no subdivision of $H$ appears as an induced subgraph in the construction of $[10,9]$ (this extended set of graphs would then provide new counterexamples to Scott's conjecture [11]). Unfortunately, our characterization is incomplete but we still provide new counterexamples to Scott's conjecture. On the other hand, we are able to give a complete characterization of all graphs $H$ that 
are $a \geqslant 2$-subdivision of some multigraph, and such that no subdivision of $H$ appears as an induced subgraph in the construction.

A consequence of Pawlik et al.'s result [10,9] is that Scott's conjecture is false for any graph obtained from $K_{5}$ by subdividing every edge at least once. We show that Scott's conjecture is also false for any graph obtained from $K_{4}$ by subdividing every edge at least once. Note that proving that Scott's conjecture holds for any subdivision of $K_{3}$ is equivalent to a long standing conjecture of Gyárfás [5], which remains open. We also prove that if $G$ is a 2-connected multigraph with no vertex intersecting every cycle of $G$, then any graph obtained from $G$ by subdividing every edge at least twice is a counterexample to Scott's conjecture. As our focus is on the construction, we do not prove Scott's conjecture is true for any particular graph, only that it cannot be proven false using the construction in some cases.

Our proof uses the following remarkable aspect of Pawlik et al.'s [9] proof (see also [6]): The graphs in the construction can be obtained not only as intersection graphs of segments in the plane, but also as intersection of a wide range of arcwise connected shapes in the plane. In this paper, we will use the fact that graphs in the construction can be represented as restricted frame graphs (see Section 2 for the definition).

Instead of focusing on the construction, we will focus on triangle-free graphs $H$ such that no subdivision of $H$ can be represented as a restricted frame graph. These subdivisions do not appear as induced subgraphs in the construction, so it follows that such graphs $H$ are counterexamples to Scott's conjecture. It turns out that graphs in the modified construction of Walczak [13] can be obtained from graphs in Pawlik et al.'s construction $[10,9,6]$ by adding twins. As the class of restricted frame graphs is stable by the operation of twin addition (see Remark 2.5), the graphs $H$ we find are also counterexamples to a weaker version of Scott's conjecture, where the chromatic number is replaced by the fractional chromatic number.

In Section 3, we characterize connected triangle-free graphs without full star cutsets (defined in that section) which are restricted frame graphs. Among other consequences, this characterization directly implies that Scott's conjecture is also false for any graph $H$ obtained from $K_{4}$ by subdividing every edge at least once. It also implies that Scott's conjecture is false for any graph $H$ which is a $\geqslant 2$-subdivision of a 2-connected multigraph $G$ with no vertex contained in all cycles. We investigate these $\geqslant 2$-subdivisions in more details in Section 4, where we show that for every multigraph $G$, either all $\geqslant 2$-subdivisions of $G$ can be represented as restricted frame graphs, or none of them can (and it can be determined in linear time whether $G$ satisfies the former or the latter property).

While it might seem restrictive to study graphs that cannot be represented as restricted frame graphs (instead of graphs that do not appear in the construction), it can be proven that in the case of $\geqslant 2$-subdivisions of multigraphs, this is not restrictive at all: for every multigraph $G$, if some $\geqslant 2$-subdivision $H$ of $G$ can be represented as a restricted frame graph, then $H$ appears as an induced subgraph in the construction of Pawlik et al. $[10,9,6]$ and in the modified construction of Walczak [13]. So the construction can be thought of as universal for $\geqslant 2$-subdivisions of restricted frame graphs. Details about the construction and this final result are given in Appendix B. 


\section{Restricted frame graphs}

As stated in the introduction, our proof relies on the analysis of the following class of graphs.

Definition 2.1. An axis-parallel box in $\mathbb{R}^{2}$ is the cartesian product of two intervals of $\mathbb{R}$. A frame is the boundary of an axis-parallel box in $\mathbb{R}^{2}$.

Definition 2.2. A graph $G$ is a restricted frame graph if it is the intersection graph of a family of frames with these restrictions (see Figure 1 for the only allowed intersection).

1. Corners of a frame do not coincide with any point of another frame,

2. the left side of any frame does not intersect any other frame,

3. if the right side of a frame intersects a second frame, this right side intersects both the top and bottom of this second frame, and

4. if two frames have non-empty intersection, then no frame is (entirely) contained in the intersection of the regions bounded by the two frames.

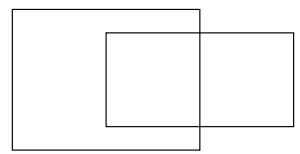

Figure 1: The only possible intersection pattern between two frames in a restricted frame graph.

A representation of a restricted frame graph $G$ is a set of frames $\mathcal{F}=\left\{F_{v} \mid v \in G\right\}$ where each $F_{v}$ is a frame and such that these frames satisfy the above restrictions and $u v \in E(G)$ if and only if $F_{u}$ intersects $F_{v}$.

We refer to $F_{v}$ as the frame of $v$ and $v$ as the vertex of $F_{v}$.

\section{Basic properties of restricted frame graphs}

Remark 2.3. As a consequence of restriction (1), when needed, we may assume all vertical sides (of all frames) occupy different $x$-coordinates and all horizontal sides (of all frames) occupy different $y$-coordinates.

Remark 2.4. A consequence of restriction (3) is that any frame which intersects the top edge of another frame also intersects the bottom edge of that frame.

Figure 1 illustrates the only way two frames are allowed to intersect in a representation of a restricted frame graph. Note that by (4), no other frame is allowed to be contained in the intersection of their interior.

Two non-adjacent vertices $u$ and $v$ of a graph $G$ are said to be twins if $N(u)=N(v)$. Remark 2.5. If a graph $G$ has a restricted frame representation and if $v$ is a vertex of $G$, the graph obtained by adding a twin of $v$ to $G$ also has a restricted frame representation (where the twin is represented by a frame just inside the frame of $v$ ). 


\section{On triangle-free restricted frame graphs}

\subsection{Basic observations and a simple subclass of triangle-free restricted frame graphs}

In this section, we characterize connected triangle-free restricted frame graphs that cannot be disconnected by the removal of the closed neighborhood of a vertex. We first describe a simple subclass of restricted frame graphs.

A graph obtained from a tree $T$ by adding a vertex $v$ adjacent to every leaf of $T$ is called a chandelier. The vertex $v$ is called the pivot of the chandelier. If the tree $T$ has the property that the neighbor of each leaf has degree two, then the chandelier is a luxury chandelier. Note that any subdivision of a (luxury) chandelier is a (luxury) chandelier.

Lemma 3.1. Any chandelier is a restricted frame graph.

Proof. Any rooted tree can be represented in such a way that the frame for the root has the leftmost left side, and the frame for leaves have the rightmost right side (see Figure 2). Now we simply add the pivot $v$ as a large box intersecting exactly these leaves frames.
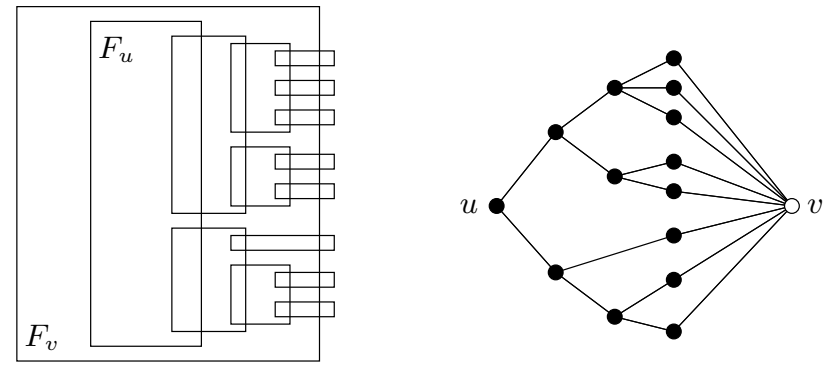

Figure 2: A frame representation of a tree $T$ rooted in $u$ together with a vertex $v$ adjacent to each of the leaves of $T$.

The remainder of this section is devoted to proving the converse of this theorem for triangle-free graphs without full star-cutsets, which we now define.

A full star-cutset in a connected graph $G$ is a set of vertices $\{u\} \cup N(u)$ whose removal disconnects $G$. The vertex $u$ is called the center of the full star-cutset and the set $\{u\} \cup$ $N(u)$, denoted by $N[u]$, is called the closed neighborhood of $u$.

Observation 3.2. A tree $T$ has no full star-cutset if and only if $T$ is a path on at most 4 vertices.

A chandelier has no full star-cutset if and only if it is a luxury chandelier.

Proof. For any tree $T$, if $T$ contains a vertex $v$ of degree at least 3 , any neighbor of $v$ is the center of a full star-cutset. Since any path with length at least 4 has a full star-cutset, $T$ is a path on at most 4 vertices.

It is easily checked that a luxury chandelier has no full star-cutset, by considering successively the cases where the deleted vertex is the vertex $v$, a leaf of the tree (this is 
where the assumption that the chandelier is luxury is used), or any other vertex of the tree.

In a chandelier that is not a luxury chandelier, some leaf $v$ of the tree has a parent of degree at least 3 and is therefore the center of a full star-cutset in the chandelier.

We now state the main result of this section, whose proof will be given in Section 3.5, after all needed lemmas.

Theorem 3.3. Suppose $H$ is a connected triangle-free graph with no full star-cutset and $H^{*}$ is a subdivision of $H$. Then $H^{*}$ is a restricted frame graph if and only if $H$ is either a path on at most 4 vertices or a luxury chandelier.

This result has the following direct consequence.

Corollary 3.4. Every connected triangle-free graph $H$ with no full star-cutset which is neither a path on at most 4 vertices, nor a luxury chandelier is a counterexample to Scott's conjecture.
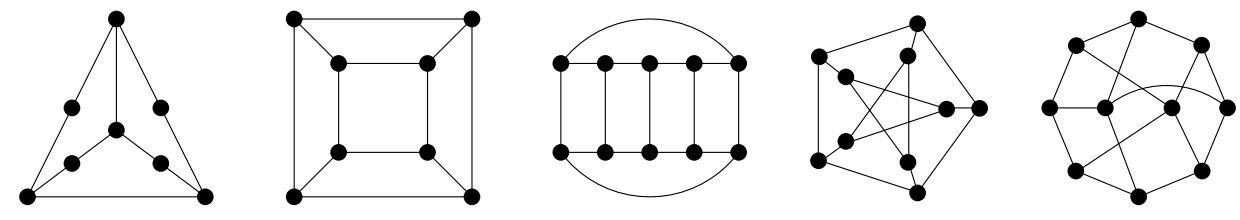

Figure 3: Some small counterexamples to Scott's conjecture.

While the condition in Theorem 3.3 (and Corollary 3.4) may seem technical, they still apply to a wide range of graphs, including small ones. For example, all the graphs of Figure 3 (as well as their subdivisions) are counterexamples to Scott's conjecture. The leftmost graph (the 1-subdivision of $K_{4}$ ), call it $H$, is particularly interesting: any trianglefree subdivision of $K_{4}$ which is not a subdivision of $H$ can be represented as a restricted frame graph (see Appendix A for details). On the other hand, such subdivisions have full star-cutsets, so this shows that the technical condition is needed in Theorem 3.3. Extensions of Corollary 3.4 remain open.

Note that cycles of length at least 5 are luxury chandeliers, so Scott's conjecture for cycles of length at least 5, which is equivalent to a conjecture of Gyárfás [5], remains open.

\subsection{Representations of restricted frame graphs}

To prove Theorem 3.3, we need some more properties of restricted frame graphs. We say that a frame $F_{1}$ contains a frame $F_{2}$ if $F_{1}$ and $F_{2}$ do not intersect and $F_{2}$ is completely contained in the region bounded by $F_{1}$. If $F_{1}$ contains $F_{2}$, we also say that $F_{2}$ is inside $F_{1}$. If $F_{1}$ and $F_{2}$ do not intersect and $F_{2}$ is not inside $F_{1}$, we say that $F_{2}$ is outside $F_{1}$. Note that if two frames intersect, none of these frames is inside another. 
Lemma 3.5 (Path Lemma). Let $\mathcal{F}=\left\{F_{v} \mid v \in G\right\}$ be a representation of a restricted frame graph $G$. For any vertices $u, v, w \in G$ where $F_{u}$ is inside $F_{v}$ and $F_{w}$ is outside $F_{v}$ and any path $P$ from $u$ to $w, P$ either contains $v$ or has an edge to $v$.

Proof. Assume that $P$ does not contain $v$. Since $F_{u}$ is inside $F_{v}$ and $F_{w}$ is outside $F_{v}$, there are two consecutive vertices $x, y$ in $P$ such that $F_{x}$ is inside $F_{v}$ and $F_{y}$ is not inside $F_{v}$. Since $F_{x}$ and $F_{y}$ intersect, $F_{y}$ contains a curve connecting a point inside $F_{v}$ and a point outside $F_{v}$. It follows from Jordan's curve theorem that $F_{y}$ intersects $F_{v}$. Hence, $y$ is adjacent to $v$ in $G$.

Corollary 3.6 (Path Corollary). For any two frames $F_{u}, F_{v}$ in a representation $\mathcal{F}$ of a restricted frame graph $G$ with $F_{u}$ inside $F_{v}$, all frames of vertices in the connected component of $G-N[v]$ containing $u$ are inside $F_{v}$.

The Path Lemma has the following important consequence.

Lemma 3.7 (Cycle Lemma). Let $\mathcal{F}=\left\{F_{v} \mid v \in G\right\}$ be a representation of a restricted frame graph $G$. For any induced cycle $C$ of $G$, there is a vertex $v \in C$ such that $F_{v}$ contains the frame of every vertex in $V(C)-N[v]$.

Moreover, if there exists another vertex $u \in C$ such that $F_{u}$ contains the frame of every vertex in $V(C)-N[u]$, then $u$ is a neighbor of $v$.

Proof. We may assume that $C$ contains at least 4 vertices, since otherwise the result holds trivially for any vertex $v \in C$. We now show how to find $v$. Among the subset $X_{C}$ of vertices of $C$ whose frame's right edge intersects the frame of another vertex of $C$, pick $v \in X_{C}$ with the largest $x$-coordinate for the right edge of its frame $F_{v}$.

We claim that the frames of the vertices of $V(C)-N[v]$ are inside $F_{v}$. To see this, let $u \in C$ be a vertex whose frame intersects the right edge of $u$, and let $w$ be the neighbor of $u$ on $C$ distinct from $v$. By the maximality of $v$, the intersection of $F_{w}$ and $F_{u}$ lies inside the region bounded by $F_{v}$ (otherwise we would have chosen $u$ or $w$ instead of $v$ ). Since $C$ contains at least 4 vertices, $w$ is not adjacent to $v$ and therefore $F_{w}$ is inside $F_{v}$. Since all elements of $V(C)-N[v]$ are in the same connected component of $G-N[v]$ as $w$, the claim follows directly from Corollary 3.6.

Assume now that there exists another vertex $u \in C$ such that $F_{u}$ contains the frame of every vertex in $V(C)-N[u]$. If $u$ and $v$ are not adjacent, then $F_{u}$ is inside $F_{v}$ and $F_{v}$ is inside $F_{u}$, a contradiction.

Given some representation $\mathcal{F}$ of $G$ as a restricted frame graph and some induced cycle $C$ of $G$, we refer to a vertex $v$ of $C$ whose frame contains every other frame of vertices of $C$ non-adjacent to $v$ as a big vertex of $C$. The frame $F_{v}$ is called a big frame of $C$. The big vertices of $\mathcal{F}$ is the set of all big vertices for all cycles of $G$. Note that these definitions depend on the chosen representation. 


\section{$3.3 \geqslant 2$-Subdivisions of $K_{4}$}

To give a flavor of the proof of Theorem 3.3, we show that no $\geqslant 2$-subdivision of $K_{4}$ is a restricted frame graph. Note that Theorem 3.3 implies the stronger result that in fact, no $\geqslant 1$-subdivision of $K_{4}$ is a restricted frame graph.

Theorem 3.8. No $\geqslant 2$-subdivision of $K_{4}$ is a restricted frame graph.

Proof. Suppose not and let $\mathcal{F}$ be a representation of a $\geqslant 2$-subdivision $G$ of $K_{4}$. By the Cycle Lemma, any subdivided cycle $C_{1}$ in $G$ has a big vertex $v_{1}$. Observe that since $G$ is a $\geqslant 2$-subdivision of $K_{4}, G-N\left[v_{1}\right]$ contains a cycle $C_{2}$ (with a big vertex $v_{2}$ ), is connected, and contains some vertex of $C_{1}$. By the Path Corollary, it follows that $F_{v_{1}}$ contains the frame of all vertices of $G-N\left[v_{1}\right]$, including $v_{2}$. By symmetry, $F_{v_{2}}$ also contains $F_{v_{1}}$, a contradiction.

\subsection{Triangle-free restricted frame graphs}

Recall that the construction of $[10,9,6]$ has no triangle, and we try to give a precise characterization of graphs which appear as induced subgraphs in the construction. Graphs that contain a triangle are clearly not contained in the construction, so we can restrict ourselves to the study of triangle-free restricted frame graphs.

Another point is that the disjoint union of two graphs $G$ and $H$ is a restricted frame graph if and only if $G$ and $H$ are restricted frame graphs. (As we shall see, induced subgraphs of the construction are also closed under taking disjoint union.) So we can also restrict ourselves to the study of connected graphs.

Lemma 3.9. Let $\mathcal{F}=\left\{F_{v} \mid v \in G\right\}$ be a representation of a connected triangle-free restricted frame graph $G$. If $G$ has no full star-cutset, the big vertices of $\mathcal{F}$ form a clique of $G$. In particular, there are at most two big vertices.

Proof. Note that by Lemma 3.7, if a cycle $C$ has two big vertices in $\mathcal{F}$, they are adjacent. Consider now two distinct cycles $C_{u}$ and $C_{v}$ of $G$ and let $u$ be a big vertex of $C_{u}$ in $\mathcal{F}$ and $v$ a big vertex of $C_{v}$ in $\mathcal{F}$, with $u \neq v$. Assume for the sake of contradiction that $u$ and $v$ are not adjacent. Since $G-N[v]$ is connected, $u$ and some (remaining) vertex of $C_{v}$ are in the same connected component of $G-N[v]$ (since $G$ is triangle-free, $C_{v}$ has length at least four and so $V\left(C_{v}\right)-N[v]$ is non-empty). By the Path Corollary, $F_{v}$ contains $F_{u}$. A symmetric argument yields that $F_{v}$ is also contained in $F_{u}$, which is a contradiction.

We conclude this subsection with two easy observations on triangle-free graphs with no full star-cutset.

Observation 3.10. Let $G$ be a connected triangle-free graph with no full star-cutset. Then $G$ has no cut-vertex of degree at least three. Moreover if $G$ is not a path with at most 4 vertices, then $G$ has minimum degree at least 2. 
Proof. Assume that $v$ is a cut-vertex of degree at least 3. If $G-v$ has more than two components, then removing the closed neighbourhood of any neighbor $u$ of $v$ removes $v$ and at most one component of $G-v$ and so $u$ is the center of a full star-cutset, a contradiction. Otherwise $G-v$ has precisely two components, and one of the two components contains at least two neighbors $u$ and $w$ of $v$, while the other contains at least one neighbor $t$ of $v$. Since $G$ is triangle-free, $u$ and $w$ are not adjacent, and so removing $N[u]$ removes $v$ and disconnects $w$ from $t$. Thus, $N[u]$ is a full star-cutset, which is a contradiction.

So $G$ contains no cut-vertex of degree at least 3 .

Now assume that $G$ is not a path with at most four vertices. If $G$ contains a vertex $x$ of degree one, let $P$ be a maximal induced path starting at $x$ and such that all internal vertices have degree two. Let $y$ be the other end of $P$. Since $G$ is neither of path with at most 4 vertices, nor a path with more than 4 vertices (such graphs contain a full starcutset), the vertex $y$ has degree at least 3 . Moreover, $y$ is a cut-vertex separating $P$ from its other neighbors, a contradiction to $G$ having no such vertex.

Observation 3.11. If $G$ is a connected triangle-free graph with no full star-cutset and $G$ is not a path on at most 4 vertices, then any subdivision $G^{*}$ of $G$ is also a (connected triangle-free) graph with no full star-cutset.

Proof. Using induction, it is enough to prove this claim when $G^{*}$ is obtained from $G$ by subdividing some edge $u v$ once, adding a new vertex $w$. Note that for any non-neighbor $x$ of $u$ in $G$ (distinct from $u$ ), if $N[x]$ is a cutset in $G^{*}$, then $N[x]$ is a cutset in $G$. Indeed, the only difference between the connected components is that in $G^{*}-N[x], w$ is added to the connected component of $u$ in $G-N[x]$. It follows that non-neighbors of $u$ (and by symmetry, non-neighbors of $v$ ) in $G$ are not centers of star-cutsets in $G^{*}$. Since $G$ is triangle-free, $u$ and $v$ have no common neighbors in $G$. Therefore, it only remains to check that $u, v$ and $w$ are not centers of full star-cutsets in $G^{*}$.

If $G^{*}-N[u]$ is disconnected then since $G-N[u]$ is connected, $G^{*}-N[u]$ has a component containing only $v$. But since $u$ and $v$ have no common neighbor in $G$, it follows that $v$ has degree one in $G$, which contradicts Observation 3.10. Hence, $u$ (and by symmetry, $v$ ) is not the center of a full star-cutset in $G^{*}$.

Finally, if $G^{*}-N[w]=G-\{u, v\}$ is disconnected then since $G-N[u]$ and $G-N[v]$ are connected, there is no vertex outside $N[u] \cup N[v]$. Since $G$ is triangle-free, it follows that $N(u)-v, N(v)-u,\{u, v\}$ form a partition of $V(G)$, and since neither $N[u]$, nor $N[v]$ is a cutset in $G$, each of these sets induces a connected subgraph in $G$. Since $G-\{u, v\}$ is disconnected, there is no edge between $N(u)-v$ and $N(v)-u$ in $G$. Therefore, $u$ and $v$ are cut-vertices in $G$ and so by Observation 3.10 they have degree at most two. Hence, $G$ is a path of length at most 4 , which is a contradiction.

\subsection{Proof of Theorem 3.3}

We are now ready to prove Theorem 3.3. To simplify the presentation, we will prove the following lemma. 
Lemma 3.12. Assume that $H$ is a connected triangle-free graph with no full star-cutset. If $H$ is a restricted frame graph then $H$ is either a path or a chandelier.

We first prove our theorem assuming this lemma.

Proof of Theorem 3.3. Let $H$ be a connected triangle-free graph with no full star-cutset, and let $H^{*}$ be a subdivision of $H$.

If $H$ is a path or a chandelier, then $H^{*}$ is also a path or a chandelier, and it follows from Lemma 3.1 that $H^{*}$ is a restricted frame graph.

Conversely, suppose $H^{*}$ is a restricted frame graph. We may assume $H$ is not a path on at most 4 vertices or we already have the desired conclusion. By Observation 3.11, $H^{*}$ is also a connected triangle-free graph with no full star-cutset. So by Lemma 3.12, $H^{*}$ is a path or a chandelier. It follows that $H$ is also a path or a chandelier. Since $H$ has no full star-cutset, by Observation $3.2, H$ is a path on at most 4 vertices or a luxury chandelier.

It remains to prove Lemma 3.12.

Proof of Lemma 3.12. Let $H$ be a connected triangle-free graph with no full star-cutset that is not a path. Assume that $H$ is a restricted frame graph, and let $\mathcal{F}=\left\{F_{v} \mid v \in H\right\}$ be a representation of $H$. By Lemma 3.9, $H$ has at most two big vertices in $\mathcal{F}$. By Observation 3.10, $H$ has minimum degree at least two. It follows that $H$ contains a cycle and therefore $H$ has at least one big vertex in $\mathcal{F}$.

If there is exactly one big vertex $u$ in $H$, then $H-\{u\}$ is a forest, and since $N[u]$ is not a cutset of $H, H-N[u]$ is a tree $T^{\prime}$. Observe that every neighbor $v$ of $u$ has exactly one neighbor in $T^{\prime}$, for if $v$ had two neighbors $v_{1}, v_{2}$ in $T^{\prime}$, then the path between $v_{1}$ and $v_{2}$ in $T^{\prime}$ together with the vertex $v$ would form a cycle in $H$ not containing $u$, contradicting the fact $H-\{u\}$ is a forest. As $H$ is triangle-free, it follows that $H-u$ is a tree $T$, and as $H$ has minimum degree 2, the leaves of $T$ are exactly the neighbors of $u$. This proves that $H$ is a chandelier, as desired.

We may now assume $H$ has precisely two big vertices $u$ and $v$ in $\mathcal{F}$. Then $u$ and $v$ are adjacent by Lemma 3.9. Since the cycle for which $u$ is big has length at least 4, $F_{u}$ contains the frame of some vertex on that cycle which is not adjacent to $u$. Since $H$ has no full star cutset, $H-N[u]$ is connected and by the Path Corollary, the frames of non-neighbors of $u$ are inside $F_{u}$ and by symmetry the frames of non-neighbors of $v$ are inside $F_{v}$. Since $F_{u}$ and $F_{v}$ intersect, by property (4) of restricted frame graphs, a vertex cannot be non-adjacent to both $u$ and $v$. Since $H$ is triangle-free, it implies that $\{u, v\}$, $N(u)-v, N(v)-u$ form a partition of $V(H)$. Since $N[v]$ is not a cutset, the subgraph induced by the neighbors of $u$ distinct from $v$ is connected. Since $N[u]$ is not a cutset, the subgraph induced by the neighbors of $v$ distinct from $u$ is connected. Since $H$ is triangle-free, each of these subgraphs is either empty or a single vertex. Since $H$ is not a path, it follows that $H$ is a cycle of length 4 , and thus $H$ is a chandelier. 


\section{$4 \geqslant 2$-Subdivisions of multigraphs}

A vertex $v$ of a graph $G$ such that $G-v$ is a forest is called a feedback vertex of $G$. We make the following remark.

Remark 4.1. If a full star-cutset in a $\geqslant 2$-subdivision of some multigraph $G$ contains a vertex $v$ of $G$, then $v$ is a cut-vertex of $G$.

Therefore, a direct consequence of Theorem 3.3 is the following:

Corollary 4.2. Let $G$ be a 2-connected multigraph with no feedback vertex. Then no $\geqslant 2$-subdivision of $G$ is a restricted frame graph.

For example, no $\geqslant 2$-subdivision of one of the graphs in Figure 4 is a restricted frame graph.
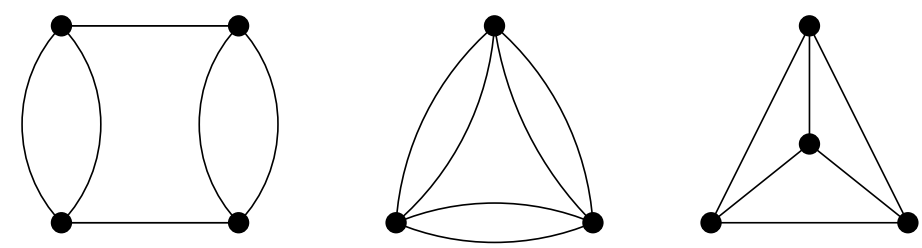

Figure 4: Scott's conjecture is false when $H$ is a $\geqslant 2$-subdivision of one of these graphs.

We now characterize all restricted frame graphs that are $a \geqslant 2$-subdivisions of some multigraphs.

Lemma 4.3. Consider a restricted frame graph $G_{1}$ and a chandelier $G_{2}$, and let $v \in G_{1}$. Then the graph $G$ obtained from the disjoint union of $G_{1}, G_{2}$ by identifying $v$ with the pivot of the chandelier $G_{2}$ is a restricted frame graph.

Proof. Let $\mathcal{F}=\left\{F_{u} \mid u \in G_{1}\right\}$ be a representation of $G_{1}$ as a restricted frame graph. By the definition of a restricted frame graph, there exists a small rectangular region $R$, whose interior contains the top right corner of $F_{v}$ and which does not intersect or is contained in any other $F_{u}$ intersecting $F_{v}$ for $u \neq v$.

Take a representation $\mathcal{D}$ of $G_{2}$ minus its pivot as a tree with all leaves on the right, such as the one depicted in Figure 2, shrink it and put $\mathcal{D}$ inside $R$, so that all leaves intersect the right side of $F_{v}$.

The union of $\mathcal{F}$ and the shrunk version of $\mathcal{D}$ is a representation of $G$.

Lemma 4.4. No $\geqslant 2$-subdivision $H^{*}$ of $\hat{H}_{1}$ or $\hat{H}_{2}$ (see Figure 5) is a restricted frame graph.

Proof. Suppose not and let $\mathcal{F}$ be a representation of $H^{*}$. Both $\hat{H}_{1}$ and $\hat{H}_{2}$ have two disjoint digons which correspond to two vertex disjoint cycles $C_{1}, C_{2}$ in $H^{*}$. Since no vertex of these digons is a cut-vertex (and no edge of the digon is a cut-edge), no vertex in $C_{i}$ is the center of a star cutset in $H^{*}$. In particular $v_{i}$, the big vertex of $C_{i}$ in $\mathcal{F}$, is not the center of a star-cutset in $H^{*}$. Thus, by the Path Corollary, $F_{v_{1}}$ is inside $F_{v_{2}}$ and $F_{v_{2}}$ is inside $F_{v_{1}}$, a contradiction. 

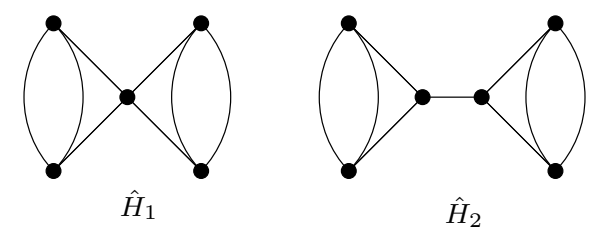

Figure 5: Graphs $\hat{H}_{1}$ and $\hat{H}_{2}$.

Given a connected graph $G$, define a bipartite graph $B_{G}=(U, V, E)$ as follows. The elements of $U$ correspond to cut-vertices of $G$, while the elements of $V$ correspond to maximal 2-connected components (also called blocks ${ }^{1}$ in the remainder) of $G$. There is an edge in $B_{G}$ between an element of $U$ and an element of $V$ if the corresponding cut-vertex belongs to the corresponding block. It is well known that $B_{G}$ is a tree, called the block decomposition, or the block tree of $G$, and that all leaves of the tree are in $V$. If one block of $G$ is set as the root of the decomposition, we obtain a rooted block decomposition of $G$. In a rooted block decomposition of $G$, the parent cut-vertex of a block of $G$ distinct from the root is defined naturally.

Corollary 4.2 and Lemmas 4.3 and 4.4 have the following consequence.

Theorem 4.5. For any connected multigraph $G$, either all $\geqslant 2$-subdivisions of $G$ are restricted frame graphs, or none of them are. Moreover, given a connected multigraph $G$, it can be determined in linear time whether $G$ satisfies the former or the latter property.

Proof. Our algorithm proceeds as follows.

1. Build the block tree of $G$. Remove leaves as long as their parent cut-vertex is a feedback vertex of the leaf.

2. If more than one block is left: answer no.

3. If one block is left: decide if it has a feedback vertex and answer yes if there is one and no otherwise. To do this, find any cycle (greedily using DFS), greedily find an ear of the cycle. Then check if any of the two vertices at the end of the ear are feedback vertices.

We now prove the correctness of the algorithm. It is enough to prove that if the algorithm answers yes for some input graph $G$, then any $\geqslant 2$-subdivision of $G$ is a restricted frame graph, while if the algorithm answers no, then no $\geqslant 2$-subdivision of $G$ is a restricted frame graph.

Assume first that the algorithm answers yes. Observe that any $\geqslant 2$-subdivision of a 2-connected multigraph with a feedback vertex $v$ is a chandelier with pivot $v$, or a path (if the multigraph is a $K_{2}$ ). It follows from the algorithm that any $\geqslant 2$-subdivision of $G$ can be obtained from a chandelier or a path by repeatedly applying the operation from

\footnotetext{
${ }^{1}$ Note that a block might consist only of two vertices of $G$ joined by an edge.
} 
Lemma 4.3 or adding a pendant vertex. This lemma implies that any $\geqslant 2$-subdivision of $G$ is a restricted frame graph.

Assume now that the algorithm answers no. So the algorithm either stopped at step (2) or (3). If the algorithm stopped at step (3), then by Corollary 4.2 no $\geqslant 2$-subdivision of $G$ is a restricted frame graph. So suppose the algorithm stopped at step (2). It follows from step (1) that in this case, the decomposition contains at least two leaves $G_{1}$ and $G_{2}$. Let $v_{1}$ and $v_{2}$ be the parent cut-vertices of $G_{1}$ and $G_{2}$. Since $v_{i}$ is not a feedback vertex of $G_{i}, G_{i}-v_{i}$ contains an (induced) cycle $C_{i}$. Moreover, since $G_{i}$ is 2-connected (and distinct from a single edge, since otherwise $v_{i}$ would be a feedback vertex of $G_{i}$ ), for any two vertices on $C_{i}$ there are internally vertex disjoint paths connecting them to $v_{i}$. Choose $C_{i}$ and these two vertices on $C_{i}$ in such a way that the maximum of the lengths of the two paths is minimized. Then add a shortest path between $v_{1}$ and $v_{2}$ in $G$ to $C_{1}, C_{2}$, and the four paths. It can be checked the subgraph induced by the vertices of these paths and cycles is isomorphic to a subdivision of either $\hat{H}_{1}$ or $\hat{H}_{2}$. It follows from Lemma 4.4 that no $\geqslant 2$-subdivision of $G$ is a restricted frame graph.

\section{Conclusion}

It was already known that any $\geqslant 1$-subdivision of a non-planar graph is a counterexample to Scott's conjecture. This recent obervation was based on the fact that a particular class of triangle-free graphs of unbounded chromatic number can be represented as intersection graphs of line segments in the plane. In this paper, we used the fact that this particular class of graphs can be represented by intersection graphs of even more specific objects in the plane, in order to provide a larger class of counterexamples. In particular, we proved that any $\geqslant 2$-subdivision of a 2-connected multigraph is a counterexample to Scott's conjecture.

This was done without studying the construction itself, only the class of intersection graphs containing it. A natural question is whether studying the construction itself would provide a larger class of couterexamples. We can show the answer is negative when we restrict ourselves to $\geqslant 2$-subdivisions of multigraphs. More details about this, as well as a description of the original construction, are given in Appendix B.

For a given graph $H$, let $\operatorname{Forb}^{*}(H)$ denote the class of graphs excluding all subdivisions of $H$ as induced subgraphs. Many special cases of the following natural refinement of Scott's conjecture remains.

Question 5.1. For which graphs $H$ is $\operatorname{Forb}^{*}(H) \chi$-bounded?

In view of Theorem 3.3 and Corollary 3.4, one such refinement seems natural.

Question 5.2. Is it true that for any luxury chandelier $G$, $\operatorname{Forb}^{*}(G)$ is $\chi$-bounded?

Note that cycles of length at least 5 are luxury chandeliers, so a positive answer to this question would imply that the class of graphs with no induced cycles on at least 5 vertices is $\chi$-bounded, which is a long-standing conjecture of Gyárfás [5].

Another special case prompted by Corollary 4.2 is the following. 
Question 5.3. For which graphs $G$ do we have that for all subdivisions $H$ of $G$, Forb* $(H)$ is $\chi$-bounded?

This we hope may have a fairly clean answer (as opposed to Question 5.1). It does not however escape the difficulty of the long-standing conjecture of Gyárfás [5] as we can pick $H$ to be a long cycle.

\section{Recent development}

After the submission of this article, there have been many exciting development. Scott and Seymour [12] have answered (in the positive) the long standing question of Gyárfás [5] stated as open here. Chudnovsky, Scott and Seymour [2] have also proved a partial converse of the result in this article.

\section{Acknowledgements}

The work at the origin of this paper was started during a workshop on $\chi$-bounded classes organized in Lyon, France, in March 2012. The authors would like to thank the organizers and participants of this meeting, in particular Feri Kardoš, Frédéric Maffray, and Stéphan Thomassé, for the discussions initiating this work. We would also like to thank the anonymous referees for their many helpful suggestions.

\section{References}

[1] J.P. Burling. On coloring problems of families of prototypes. PhD thesis, University of Colorado, Boulder, 1965.

[2] M. Chudnovsky, A. Scott and P. Seymour. Induced subgraphs of graphs with large chromatic number. V. Chandeliers and strings. Manuscript

[3] P. Erdős. Graph theory and probability. Canadian Journal of Mathematics, 11:34-38, 1959.

[4] A. Gyárfás. On Ramsey covering numbers. Infinite and Finite Sets, Coll. Math. Soc. J. Bolyai, 10:801-816, 1973.

[5] A. Gyárfás. Problems from the world surrounding perfect graphs. Applicationes Mathematicae, 19(3-4):413-441, 1987.

[6] T. Krawczyk, A. Pawlik, and B. Walczak. Coloring triangle-free rectangle overlap graphs with $O(\log \log n)$ colors. Discrete $\&$ Computational Geometry, 53(1):199-220, 2015.

[7] B. Lévêque, F. Maffray, and N. Trotignon. On graphs with no induced subdivision of $K_{4}$. Journal of Combinatorial Theory, Series B, 102(4):924-947, 2012.

[8] J. Mycielski. Sur le coloriage des graphes. In Colloq. Math, volume 3, pages 161-162, 1955. 
[9] A. Pawlik, J. Kozik, T. Krawczyk, M. Lasoń, P. Micek, W.T. Trotter, and B. Walczak. Triangle-free geometric intersection graphs with large chromatic number. Discrete \& Computational Geometry, 50(3):714-726, 2013.

[10] A. Pawlik, J. Kozik, T. Krawczyk, M. Lasoń, P. Micek, W.T. Trotter, and B. Walczak. Triangle-free intersection graphs of line segments with large chromatic number. Journal of Combinatorial Theory, Series B, 105:6-10, 2014.

[11] A. Scott. Induced trees in graphs of large chromatic number. Journal of Graph Theory, 24(4):297-311, 1997.

[12] A. Scott and P. Seymour Induced subgraphs of graphs with large chromatic number. I. Odd holes Journal of Combinatorial Theory, Series B, in press, 2015.

[13] B. Walczak. Triangle-free geometric intersection graphs with no large independent sets. Discrete $\&$ Computational Geometry, 53(1):221-225, 2015. 


\section{A Subdivisions of $\boldsymbol{K}_{4}$}

It was proved by Scott (see [7]) that there exists a constant $c$ such that graphs with no induced subdivisions of $K_{4}$ have chromatic number at most $c$. It remains interesting to understand which subdivisions of $K_{4}$ are responsible for this bound on the chromatic number.

The type of a subdivision of $K_{4}$ is the number of subdivided edges in the original copy of $K_{4}$. For instance, a type 6 subdivision of $K_{4}$ is obtained from $K_{4}$ by subdividing each edge at least once, while a type 0 subdivision of $K_{4}$ is just a copy of $K_{4}$.

Corollary 3.4 directly implies that any type 6 or type 5 subdivision of $K_{4}$, and any type 4 subdivision of $K_{4}$ in which the non-subdivided edges of $K_{4}$ do not share a vertex are counterexamples to Scott's conjecture. In this section, we show that every other triangle-free subdivision of $K_{4}$ can be represented as a restricted frame graph.

In Figure 6, we show that starting from a frame representation of a graph $G$, and given a specific edge $u v$ of $G$, we can inductively construct frame representations of graphs obtained from $G$ by subdividing the edge $u v$. These operations are only valid if the intersection of the frames of $u$ and $v$ in the original representation of $G$ is of a certain type. We omit the details, since it can be easily checked that these operations work fine in the representations of the graphs of Figure 7.
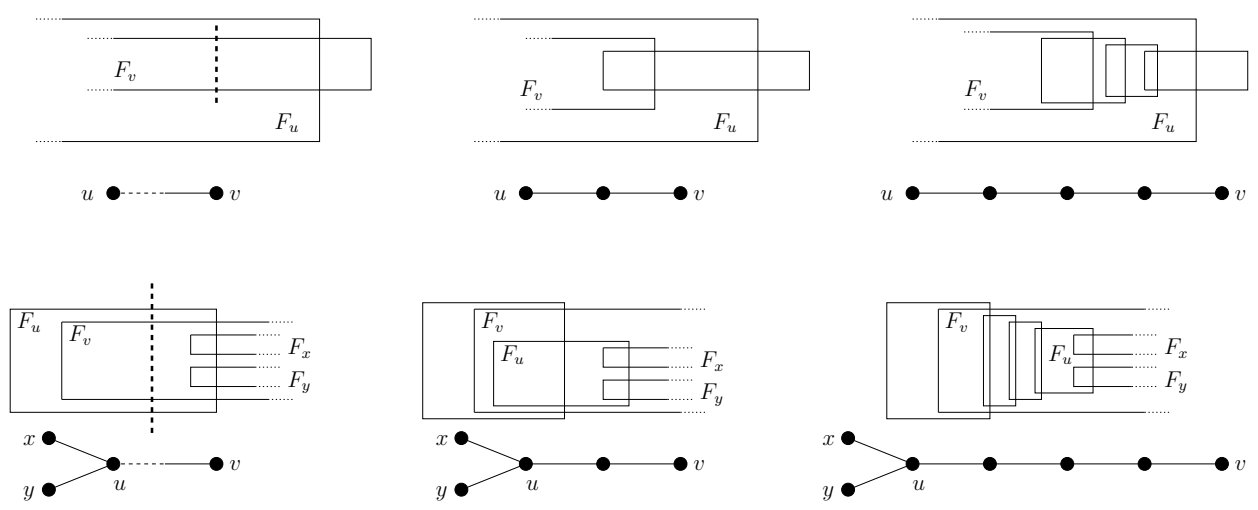

Figure 6: A vertical dashed line corresponds to an intersection we replace by (a path of) frames, so that given a frame representation of the original graph, we can deduce a frame representation of any graph obtained by subdividing the edge $u v$.

We can now state our result about triangle-free subdivisions of $K_{4}$.

Theorem A.1. Let $G$ be a triangle-free subdivision of $K_{4}$. Then $G$ is a restricted frame graphs if and only if

1. $G$ is $K_{4}$ with at most 3 of the 6 edges subdivided, or

2. $G$ is $K_{4}$ with 4 subdivided edges and the two non-subdivided edges share a vertex. 

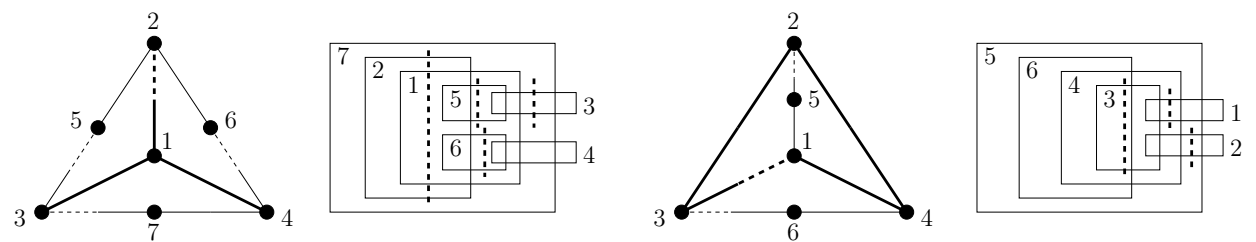

Figure 7: Frame representations of triangle-free subdivisions of $K_{4}$ that are restricted frame graphs.

Proof. From Corollary 3.4, we know that any type 6 or type 5 subdivision of $K_{4}$, and any type 4 subdivision of $K_{4}$ in which the non-subdivided edges of $K_{4}$ do not share a vertex cannot be a restricted frame graph.

For the other triangle-free subdivisions of $K_{4}$, the construction is given in Figure 7 . The convention on the figure is the following: vertical dashed lines correspond to intersections we replace by (a path of) frames according to Figure 6 .

For the graph on the left of Figure 7, we can subdivide the edges 35 and 46 as depicted in Figure 6 (top), the edge 37 as depicted in Figure 6 (middle), and the edge 12 as depicted in Figure 6 (bottom).

For the graph on the right of Figure 7, we can subdivide the edge 13 as depicted in Figure 6 (top), the edge 25 as depicted in Figure 6 (middle), and the edge 46 as depicted in Figure 6 (bottom).

It can be checked that for these two graphs, the introduction of (paths of) frames as depicted in Figure 6 yields representations satisfying (1)-(4) in Definition 2.2, therefore all the subdivisions of $K_{4}$ considered here are restricted frame graphs.

\section{B The construction}

Our ultimate goal is to characterize (multi)graphs $G$ such that all $\geqslant 2$ subdivisions of $G$ appear as an induced subgraph in the construction of [9]. But in the previous sections, we instead characterized (multi)graphs $G$ where all $\geqslant 2$ subdivisions of $G$ are restricted frame graphs (Theorem 4.5), which at first seems more restrictive. In this section, we bridge this gap by showing that the two classes are in fact the same.

We first show that any graph appearing as an induced subgraph in the construction of [9] can be obtained by repeatedly applying two fairly simple operations, ADD and JOIN. We then deduce that restricted frame graphs that are $\geqslant 2$-subdivisions of some multigraph appear as an induced subgraph in the construction (Theorem B.4).

Definition B.1. A graph-stable set pair $(G, \mathcal{S})$ is a graph $G$ together with a set $\mathcal{S}$ of stable sets of $G$.

A graph-stable set pair $(G, \mathcal{S})$ is an induced subgraph-stable set pair of $\left(H, \mathcal{S}^{\prime}\right)$ if $G$ is an induced subgraph of $H$ and $\mathcal{S}$ is a subset of the restriction of $\mathcal{S}^{\prime}$ to the vertices of $G$.

We define an iterative process which yields exactly the graphs in Burling and Pawlik et al.'s construction. 
Definition B.2. We define a procedure NEXT which takes as input a graph-stable set pair $(G, \mathcal{S})$ and returns a graph-stable set pair $\left(G^{\prime}, \mathcal{S}^{\prime}\right) .\left(G^{\prime}, \mathcal{S}^{\prime}\right)$ is obtained from $(G, \mathcal{S})$ by

1. adding $|\mathcal{S}|$ disjoint copies $\left(H_{S}, \mathcal{S}\left(H_{S}\right)\right)$ of $(G, \mathcal{S})$, indexed by stable sets $S \in \mathcal{S}$,

2. adding a vertex $v_{S, T}$ whose neighborhood is exactly $T$ for each $S \in \mathcal{S}$ and for each $T \in \mathcal{S}\left(H_{S}\right)$, and

3. setting $\mathcal{S}^{\prime}$ as the union of $\left\{S \cup T \mid S \in \mathcal{S}, T \in \mathcal{S}\left(H_{S}\right)\right\}$ and $\left\{S \cup\left\{v_{S, T}\right\} \mid S \in \mathcal{S}, T \in\right.$ $\left.\mathcal{S}\left(H_{S}\right)\right\}$.
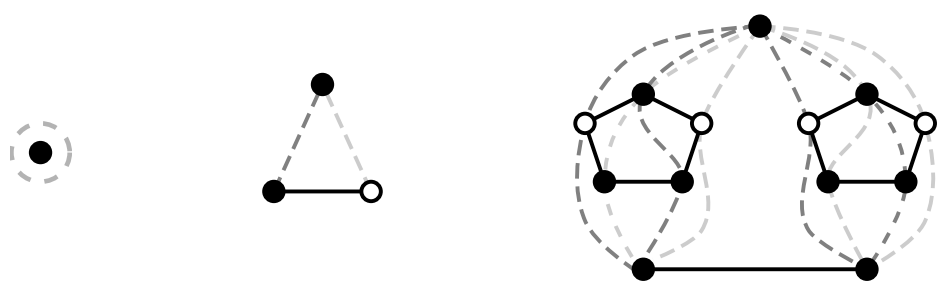

Figure 8: The graph-stable set pairs from the first 3 steps of the construction. Vertices $v_{S, T}$ are white and each stable set in $S \in \mathcal{S}$ is a dashed line through the elements of $S$.

Definition B.3. We say that a graph-stable set pair $(G, \mathcal{S})$ is constructible if it is an induced subgraph-stable set pair of $\operatorname{NEXT}^{i}\left(G_{0}, \mathcal{S}_{0}\right)$ for some $i$, where $G_{0}=K_{1}$, $\mathcal{S}_{0}=$ $\left\{V\left(G_{0}\right)\right\}$.

We drew the first few graph-stable set pairs $\operatorname{NEXT}^{j}\left(G_{0}, \mathcal{S}_{0}\right)$ in Figure 8 and it is not too difficult to check that the graph of $\operatorname{NEXT}^{j}\left(G_{0}, \mathcal{S}_{0}\right)$ is the $j$ th graph of Burling and Pawlik et al.'s construction for each $j$. We can now state this section's main result.

Theorem B.4. For every restricted frame graph $H$ that is also a $\geqslant 2$-subdivision of some multigraph, there is a subset $\mathcal{S}$ of stable sets of $H$ for which $(H, \mathcal{S})$ is constructible.

As a consequence, any restricted frame graph $H$ that is also a $\geqslant 2$-subdivision (of some multigraph) appears as an induced subgraph of the construction.

To prove Theorem B.4, we use two simple operations that preserve constructability rather than NEXT.

Definition B.5. By adding a vertex $v$ on $S$ to a graph-stable set pair $(G, \mathcal{S})$ with $S \in \mathcal{S}$, we mean to build a new graph-stable set pair $\left(H, \mathcal{S}^{\prime}\right)$ where $H$ is $G$ with a new vertex labelled $v$ whose neighborhood is $S$ and $\mathcal{S}^{\prime}=\mathcal{S} \cup\{\{v\}\}$.

We define the function $\operatorname{ADD}$ as $\operatorname{ADD}((G, \mathcal{S}), S)=\left(H, \mathcal{S}^{\prime}\right)$.

By joining a graph-stable set pair $\left(G_{1}, \mathcal{S}_{1}\right)$ to a graph-stable set pair $\left(G_{2}, \mathcal{S}_{2}\right)$ on $S \in \mathcal{S}_{2}$, we mean to build a graph-stable set pair $\left(H, \mathcal{S}^{\prime}\right)$ where $H$ is the disjoint union of $G_{1}$ and $G_{2}$ and $\mathcal{S}^{\prime}=\left(\mathcal{S}_{2}-\{S\}\right) \cup\left\{S \cup S_{1} \mid S_{1} \in \mathcal{S}_{1}\right\}$.

We define the function JOIN as $\operatorname{JOIN}\left(\left(G_{1}, \mathcal{S}_{1}\right),\left(G_{2}, \mathcal{S}_{2}\right), S\right)=\left(H, \mathcal{S}^{\prime}\right)$. 
In other words, the operation ADD adds a vertex to the graph adjacent to all vertices of a specific stable set and then adds a stable set containing only this new vertex. The second operation is the disjoint union of two graphs and the elements of a specific stable set in the second graph-stable set pair are added to all stable sets of the first graph-stable set pair.

To simplify the discussion, we allow joining on the empty stable set (which results in the disjoint union of the graph and the disjoint union of the stable sets). This can be simulated by adding a vertex $v$ using ADD, joining on $\{v\}$, and then removing $v$ (by taking an induced subgraph-stable set pair).

The following observations tell us that applying these two operations to constructible graph-stable set pairs yields a constructible graph-stable set pair.

Observation B.6. $\operatorname{ADD}((G, \mathcal{S}), S)$ is an induced subgraph-stable set pair of $\operatorname{NEXT}(G, \mathcal{S})$ for any $S \in \mathcal{S}$.

Proof. To see this, take any $T \in \mathcal{S}$ and note that $v_{T, S}$ has the desired neighborhood in the subgraph of the graph of $\operatorname{NexT}(G, \mathcal{S})$ induced by $V\left(G_{T}\right) \cup\left\{v_{T, S}\right\}$.

Moreover by definition of $\operatorname{NEXT}(G, \mathcal{S})$, the stable sets in the graph-stable set pair of $\operatorname{NEXT}(G, \mathcal{S})$ induced by $V\left(G_{T}\right) \cup\left\{v_{T, S}\right\}$ are precisely $\mathcal{S} \cup\left\{v_{T, S}\right\}$, as desired.

Observation B.7. If $\left(G_{1}, \mathcal{S}_{1}\right)$ and $\left(G_{2}, \mathcal{S}_{2}\right)$ are both induced subgraph-stable set pairs of $(H, \mathcal{S})$, then for any $S \in \mathcal{S}_{2}$, JOIN $\left(\left(G_{1}, \mathcal{S}_{1}\right),\left(G_{2}, \mathcal{S}_{2}\right), S\right)$ is an induced subgraph-stable set pair of $\operatorname{NEXT}(H, \mathcal{S})$.

Proof. To see this, consider $\operatorname{Next}(H, \mathcal{S})$ : the original copy of $(H, \mathcal{S})$ contains an induced copy of $\left(G_{2}, \mathcal{S}_{2}\right)$, and the new copy $\left(H_{S}, \mathcal{S}\left(H_{S}\right)\right)$ contains an induced copy of $\left(G_{1}, \mathcal{S}_{1}\right)$.

There exists $S^{\prime} \in \mathcal{S}$ such that $S=S^{\prime} \cap V\left(G_{2}\right)$ and for each $S_{1} \in \mathcal{S}_{1}$, there exists $S_{1}^{\prime} \in \mathcal{S}$ such that $S_{1}=S_{1}^{\prime} \cap V\left(G_{1}\right)$. Note that $S^{\prime} \cup S_{1}^{\prime}$ is a stable set of $\operatorname{NEXT}(H, \mathcal{S})$, and that $S \cup S_{1}=\left(S^{\prime} \cup S_{1}^{\prime}\right) \cap\left(V\left(G_{1}\right) \cup V\left(G_{2}\right)\right)$. Moreover, for each $S_{2} \in \mathcal{S}_{2}-\{S\}$, there exists $S_{2}^{\prime} \in \mathcal{S}$ such that $S_{2}=S_{2}^{\prime} \cap V\left(G_{2}\right)$. Note that $S_{2}^{\prime} \cup\left\{v_{S_{2}^{\prime}, S^{\prime}}\right\}$ is a stable set of $\operatorname{NEXT}(H, \mathcal{S})$, and that $S_{2}=\left(S_{2}^{\prime} \cup\left\{v_{S_{2}^{\prime}, S^{\prime}}\right\}\right) \cap\left(V\left(G_{1}\right) \cup V\left(G_{2}\right)\right)$.

Consequently, $\operatorname{JOIN}\left(\left(G_{1}, \mathcal{S}_{1}\right),\left(G_{2}, \mathcal{S}_{2}\right), S\right)$ is an induced subgraph-stable set pair of $\operatorname{NEXT}(H, \mathcal{S})$.

We sum up the previous observations into the following remark.

Remark B.8. If $\left(G_{1}, \mathcal{S}_{1}\right),\left(G_{2}, \mathcal{S}_{2}\right)$ are constructible graph-stable set pairs, then for any $S \in \mathcal{S}_{2}, \operatorname{ADD}\left(\left(G_{2}, \mathcal{S}_{2}\right), S\right)$ and JOIN $\left(\left(G_{1}, \mathcal{S}_{1}\right),\left(G_{2}, \mathcal{S}_{2}\right), S\right)$ are constructible graph-stable set pairs.

ADD's preservation of constructability has the following easy consequence.

Lemma B.9. For any tree $T,(T,\{\{u\} \mid u \in T\})$ is a constructible graph-stable set pair.

Proof. Start with the singleton graph-stable set pair and repeatedly apply ADD to build $T$. 
We will now need the following decomposition result, which is a direct consequence of the proof of Theorem 4.5.

Corollary B.10. For any connected multigraph $G$ such that some $\geqslant 2$-subdivision $H$ of $G$ is a restricted frame graph, $G$ has a rooted block decomposition where the root block has a feedback vertex, and for each block $B$ distinct from the root block, the parent cut-vertex of $B$ is a feedback vertex of $B$.

This decomposition of $G$ induces a decomposition of $H$ with the same properties. We insist on the fact that this decomposition of $H$ is not a block decomposition as defined earlier, since a block consisting of a single edge in $G$ corresponds to a block consisting of a path on at least 3 edges in $H$ (such a path is not 2-connected). However, any block distinct from a single edge in $G$ corresponds to a 2-connected block in $H$. We will refer to this decomposition of $H$ as a pseudo-decomposition, and the $\geqslant 2$-subdivision of each block of $G$ will be called a pseudo-block of $H$.

Note that this pseudo-decomposition has the additional property that any cut-vertex of $G$ distinct from $r$ (the feedback vertex of the root block) is at distance at least 3 from $r$ in $H$. In what follows, $r$ will be simply called the root of $H$.

Our proof of Theorem B.4 uses the following technical lemma which can be thought of as a strenghening of Theorem B.4 that is better adapted for a proof by induction.

Lemma B.11. Let $H$ be a connected restricted frame graph that is a $\geqslant 2$-subdivision of some multigraph $G$, and let $r$ be the root of $H$. Then there is a set $\mathcal{S}$ of stable sets of $H-N[r]$ containing all singletons $\{v\}$, where $v$ is at distance 2 from $r$ in $H$, such that the graph-stable set pair $(H-N[r], \mathcal{S})$ is constructible.

Proof. We prove the result by induction on the number of vertices of $H$. If $H$ is 2connected then $H-N[r]$ is a tree and the result follows from Lemma B.9. Otherwise, for any cut-vertex $s$ of $G$ lying in the root block $R$, let $R, H_{s}^{1}, \ldots, H_{s}^{k}$ be the subgraphs of $H$ induced by the vertex-set of each component of $H-s$ together with $s$. By induction, for each $s$ and each $i$, there is a set $\mathcal{S}_{s}^{i}$ of stable sets of $H_{s}^{i}-N[s]$ including all singletons $\{v\}$, where $v$ is at distance 2 from $s$ in $H_{s}^{i}$, and such that $\left(H_{s}^{i}-N[s], \mathcal{S}_{s}^{i}\right)$ is constructible. Consequently, it follows from the fact that the disjoint union of two constructible graphstable set pairs is a constructible graph-stable set pair (see the remark above on joining on the empty set), that for any $s$, the disjoint union of all $\left(H_{s}^{i}-N[s], \mathcal{S}_{s}^{i}\right), i \geqslant 1$, forms a constructible graph-stable set pair. We will refer to this graph-stable set pair as $\left(H_{s}, \mathcal{S}_{s}\right)$.

By Lemma B.9, $H_{0}=R-N[r]$ together with the set $\mathcal{S}_{0}=\{\{v\} \mid v \in R-N[r]\}$ is a constructible graph-stable set pair. Let $s_{1}, \ldots, s_{\ell}$ be the cut-vertices of $G$ lying in $R-r$. We define two graphs $G_{i}$ and $H_{i}$ and a family of stable sets $\mathcal{S}_{i}$ iteratively as follows. For $i=1 \ldots \ell$, let $\left(G_{i}, \mathcal{S}_{i}\right)$ be obtained by joining $\left(H_{s_{i}}, \mathcal{S}_{s_{i}}\right)$ and $\left(H_{i-1}, \mathcal{S}_{i-1}\right)$ on $\left\{s_{i}\right\}$. This JOIN operation creates stable sets $\left\{s_{i}, u\right\}$ for all vertices $u$ at distance 2 from $s_{i}$ in $H_{s_{i}}$ (while $\mathcal{S}_{i}$ still contains singletons $\left\{s_{j}\right\}$ for any $j>i$ ). Let $H_{i}$ be obtained from $G_{i}$ by adding, for each such pair $\left\{s_{i}, u\right\}$ a new vertex adjacent to $s_{i}$ and $u$. We now define a graph-stable set pair $\left(H^{\prime}, \mathcal{S}^{\prime}\right)$ as follows: if $r$ is not a cut-vertex in $G$, then $\left(H^{\prime}, \mathcal{S}^{\prime}\right)=\left(H_{\ell}, \mathcal{S}_{\ell}\right)$, and 
otherwise $\left(H^{\prime}, \mathcal{S}^{\prime}\right)$ is the graph-stable set pair obtained by taking the disjoint union of $\left(H_{\ell}, \mathcal{S}_{\ell}\right)$ and $\left(H_{r}, \mathcal{S}_{r}\right)$ (i.e., joining them on the empty set).

It follows from the fact that $H$ is a $\geqslant 2$-subdivision of some multigraph, that $H^{\prime}$ is precisely $H-N[r]$ and $\mathcal{S}^{\prime}$ has the desired property (since vertices at distance two from $r$ in $H$ are not cut-vertices).

It remains to prove Theorem B.4.

Proof of Theorem B.4. Observe that $(H, \mathcal{S})$ is constructible for some set $\mathcal{S}$ of stable sets of $H$ if and only if all its connected components are. Hence, it is enough to prove the theorem when $H$ is connected. In this case, by Lemma B.11, there is a set $\mathcal{S}^{\prime}$ of stable sets of $H-N[r]$, where $r$ is the feedback vertex of the root pseudo-block of the pseudo decomposition, such that $\mathcal{S}^{\prime}$ contains all singletons $\{v\}$ where $v$ is at distance two from $r$ in $H$. Let $H_{r}$ consist of a single vertex $r$, and $\mathcal{S}_{r}=\{\{r\}\}$. We join the constructible graph-stable set pair $\left(H-N[r], \mathcal{S}^{\prime}\right)$ and $\left(H_{r}, \mathcal{S}_{r}\right)$ on $\{r\}$, and the obtained graph-stable set pair contains pairs $\{u, r\}$ for any $u$ is at distance two from $r$ in $H$. The graph obtained by adding a vertex adjacent to $u$ and $r$, for every such pair, is precisely $H$.

Combining Theorems 4.5 and B.4, and the discussion about joining on the empty set, we obtain the following immediate corollary.

Corollary B.12. For any multigraph $G$, either all $\geqslant 2$-subdivisions of $G$ appear as induced subgraphs in the construction of Burling and Pawlik et al., or none of them do. 